\title{
Factores determinantes del abandono del Programa de Hipertensión Arterial. Hospital Nacional “Almanzor Aguinaga Asenjo” EsSalud, Chiclayo 2000
}

\author{
VÍCTOR SOTO, SEGUNDO ZAVALETA, JOSÉ BERNILLA \\ Oficina de Epidemiología y Programas de Salud HNAAA, Hospital Nacional Almanzor Aguinaga
}

\begin{abstract}
RESUMEN
OBJETIVOS: Precisar los factores determinantes por los que los pacientes asegurados abandonan el Programa de Hipertensión Arterial. LUGAR: Hospital Nacional Almanzor Aguinaga de Chiclayo. Diseño: Estudio epidemiológico analítico de casos y controles. MATERIAL Y MÉTODOS: Los casos fueron 126 pacientes que abandonaron el Programa por más de seis meses y los 252 controles los que continuaban en el mismo, pareados según sexo, edad, procedencia y estadío de hipertensión; excluyéndose los que tuvieron complicaciones o cambios de adscripción. Se aplicó una encuesta a domicilio. RESULTADOS: El factor conocimiento inadecuado de la enfermedad tuvo un OR de 15,3, seguido del desinterés en el control mensual (OR: 14,6), incumplimiento del tratamiento por normalización de las cifras de presión arterial (OR: 13,8) o incumplimiento por no tener molestias (OR: 13,2), los que en conjunto tienen que ver con una conciencia inapropiada de la enfermedad. Otros factores fueron: inalterabilidad del estilo de vida (OR: 4,5) y efectos adversos del medicamento (OR: 1,9), no encontrándose asociación con el maltrato del profesional asistencial, hacinamiento, incomodidad, horarios inadecuados o problemas económicos o de traslado del paciente. CONCLUSIÓN: El conocimiento inadecuado sobre la hipertensión arterial fue el principal factor determinante del abandono del Programa de Hipertensión Arterial en el Hospital Nacional Almanzor Aguinaga Asenjo de Essalud.
\end{abstract}

Palabras clave: Hipertensión; programas nacionales de salud; educación del paciente; negativa del paciente al tratamiento.

\begin{abstract}
DETERMINING FACTORS FOR ARTERIAL HYPERTENSION PROGRAM DESERTION. CHICLAYO ALMANZOR AGUINAGA ASENJO NATIONAL HOSPITAL, ESSALUD 2000 SUMMARY

OBJETIVES: To determine the main factors for desertion from the Arterial Hypertension Program. SETTING: Chiclayo Almanzor Aguinaga National Hospital. DESIGN: Analytical case-control study. MATERIAL AND METHODS: Cases were 126 patients who deserted for over 6 months and controls the 252 patients who continued in the program, paired for sex, age, place of referral and hypertension level; patients with complications or adscription changes were excluded. A domiciliary survey was applied. RESULTS: Low knowledge about hypertension was the main factor (OR: 15.31), including desinterest in monthly control (OR: 14,6), treatment negligence when both arterial blood pressure returned to normality (OR: 13,8) or when asymptomatic (OR: 13,2), all meaning inappropiate illness consciousness. Other factors found were: No change in life style (OR: 4,5), and colateral drug effects (OR: 1,9); there was no association with professional mistreatment, overcrowding, discomfort, inadequate schedule or patient's economical or translation problems. CONCLUSION: Inadequate knowledge on arterial hypertension was de determinant factor for Arterial Hypertension Program desertion at EsSalud Almanzor Aguinaga Asenjo National Hospital.
\end{abstract}

Key words: Hypertension; national health programs; patient education; treatment refusal.

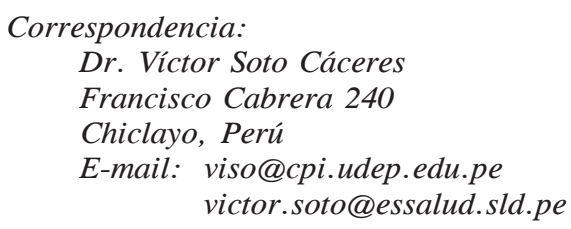




\section{INTRODUCCIÓN}

Entre las enfermedades crónicas no transmisibles, destaca en la actualidad la hipertensión arterial, tanto a nivel nacional como internacional, siendo su magnitud tal que el Seguro Social del Perú (hoy EsSalud) instauró un Programa de Control de Hipertensión Arterial en todos sus establecimientos, dentro de los denominados Programas Externos (antes Programas Preventivo Promocionales) $\left({ }^{1}\right)$.

A partir de la década de los 80 , la muerte por enfermedad del corazón ha variado paulatinamente su contribución proporcional en la población adulta; así tenemos que en los países desarrollados ha descendido, mientras que en los en vías de desarrollo ha incrementado, ubicándose dentro de las tres principales causas de mortalidad $\left({ }^{2}\right)$.

Por otro lado, la hipertensión arterial está considerada como la enfermedad crónica más frecuente de la humanidad $\left({ }^{3}\right)$. De manera general, se estima que entre 10 y $30 \%$ de la población mundial adulta tiene cifras de presión arterial superior a los niveles convencionales $\left({ }^{4,5}\right)$.

La prevalencia de hipertensión arterial en nuestro medio ha sido estudiada por varios investigadores, entre ellos Oliveros, que encontró una prevalencia media de $20 \%$, indicando diversas cifras relativas al conocimiento, tratamiento y control de la enfermedad, según el lugar donde se manejan los casos. El de mayor captación respecto a su población es el Seguro Social $\left({ }^{6}\right)$. Soto Cáceres y colaboradores determinaron la prevalencia de hipertensión en la población adulta de Chiclayo, mediante estudio poblacional domiciliario, encontrando $26,6 \%$ de incidencia, siendo de estadio I $17,4 \%$, II $6 \%$, III $2,5 \%$ y IV $0,7 \%$, existiendo además asociación con la obesidad $\left({ }^{7}\right)$; mientras que, en los asegurados la cifra proporcionalmente fue más alta que en la población general $\left({ }^{8}\right)$.

También en otros lugares vecinos a Chiclayo se estudió la prevalencia de hipertensión arterial, como en Monsefú, siendo 10\%, sin diferencia por sexos. Sólo $47 \%$ conocía su situación y so- lamente $14 \%$ llevaba un tratamiento antihipertensivo con regularidad $\left({ }^{9}\right)$.

La mayoría de los casos de hipertensión arterial no tiene una causa definida, por lo que se le denomina hipertensión esencial. Pero, se considera que participan en ella diversos factores, como herencia, edad, raza, tabaco, falta de ejercicio, obesidad, estrés crónico y exceso de sal en la dieta. La presencia de uno o más factores incrementa la posibilidad de desarrollar, mantener o agravar la enfermedad $\left({ }^{10-12}\right)$.

Existe amplia evidencia de que la morbilidad y mortalidad cardiovascular en relación con la hipertensión arterial pueden reducirse con un tratamiento antihipertensivo adecuado $\left({ }^{13}\right)$, tal como se viene prescribiendo en base a protocolo en el Seguro Social $\left({ }^{14}\right)$. Obviamente, es necesario que el cumplimiento del tratamiento sea el indicado para lograr el éxito esperado.

En EsSalud existe un programa específico de control de hipertensión arterial y los asegurados tienen acceso en forma gratuita al medicamento y con facilidades en el sistema de citas, recibiendo atención médica cada 3 meses (4 veces al año) y atención de enfermería en los meses intermedios, en espera del nuevo control médico ( 8 veces al año). En todos los controles se verifica la cifra de presión arterial y se hace entrega de las recetas de medicamentos específicos. Existe una alta cifra de pacientes registrados, cercano a los 3,000 en el Hospital Nacional Almanzor Aguinaga (HNAA), aunque una buena proporción ha sido reubicada recientemente por lugar de adscripción $\left({ }^{15}\right)$.

Sin embargo, a pesar de las aparentes facilidades que tienen los pacientes asegurados, existe un abandono preocupante del Programa, estimándose en alrededor de 10 a $15 \%$ anual $\left({ }^{16}\right)$.

En consecuencia, es de importancia averiguar por qué se presenta este alto índice de deserción de un Programa de enfermedad crónica, lo que permitirá plantear estrategias para corregir este aspecto y evitar las complicaciones y muerte por la enfermedad. 
Los objetivos de la presente investigación fueron:

1. Precisar los factores determinantes de abandono del Programa de Hipertensión Arterial del HNAA.

2. Verificar si el conocimiento inadecuado de la enfermedad, desconfianza en el personal de salud o la conciencia inapropiada de su enfermedad o la situación económica del pacientes son factores determinantes del abandono del Programa.

3. Determinar si factores relacionados con los medicamentos y sus efectos colaterales, o aspectos administrativos relacionados con infraestructura u horarios son factores de abandono del Programa.

\section{MATERIAL Y MÉTODOS}

El tipo de investigación fue analítico de casos y controles. La población fue la totalidad de pacientes registrados en el Programa de Hipertensión Arterial del HNAA, residentes en la ciudad de Chiclayo, con estadio de hipertensión II ó III. Los casos fueron los pacientes registrados que habían abandonado el Programa por un período mayor de seis meses, mayores de 50 años de edad, estadio II ó III $\left({ }^{17,18}\right)$. Y los controles, pacientes que no abandonaron el Programa, continuando su tratamiento en forma mensual. Se equiparó ambos grupos por sexo, edad, estadio de hipertensión arterial y similar tiempo de ingreso al Programa.

Se excluyó a los pacientes que cambiaron de establecimiento de adscripción, estuvieron en otros servicios hospitalarios por complicaciones, o habían fallecido.

Para el cálculo de la muestra se utilizó la fórmula correspondiente de tamaño de muestra, con un nivel de confianza del $95 \%$ (Z) y un error del $5 \%(\mathrm{~T})$, considerando una proporción de abandono en el último semestre de 9\% (P).

Se consideró casos a 126 pacientes y se optó por tomar dos pacientes controles por cada caso, es decir, 252 controles, ubicándose los pacientes según los registros y fichas de control existentes en el Programa.

Como instrumento se utilizó una encuesta tipo entrevista estructurada aplicada por los investigadores a los pacientes casos y controles seleccionados, la que se realizó en el domicilio a los casos (abandonos) y en el Programa de HTA a los controles; el tiempo de cada entrevista fue 15 minutos promedio.

Para el análisis de datos, se aplicó el enfoque de riesgo en casos y controles, mediante el odds ratio $(\mathrm{OR})$ o prueba de factores cruzados.

\section{RESULTADOS}

El factor conocimiento inadecuado sobre la enfermedad (definido como respuestas erradas sobre complicaciones, duración de la enfermedad) presentó un OR de 15,3 (Tabla 1).

El factor desinterés en el control mensual en el Programa fue el segundo en importancia, con un OR de 14,6 (Tabla 2), seguido del incumplimiento del tratamiento debido a la normalización de las cifras de la presión arterial,con OR 13,8 (Tabla 3). Otro factor relacionado con el incumplimiento del tratamiento antihipertensivo fue el ya no sentir molestias, con OR 13,2 (Tabla 4). Estos aspectos, en conjunto, se refieren a que los pacientes tienen una conciencia inapropiada de la enfermedad.

Tabla 1.- Distribución de casos y controles según factor conocimiento inadecuado sobre la enfermedad.

\begin{tabular}{|c|c|c|c|}
\hline \multirow[b]{2}{*}{ Factor } & \multicolumn{2}{|c|}{ Abandono } & \multirow[b]{2}{*}{ Total } \\
\hline & $\overline{\text { Sí }}$ & No & \\
\hline Positivo & 62 & 15 & 77 \\
\hline Negativo & 64 & 237 & 301 \\
\hline Total & 126 & 252 & 378 \\
\hline
\end{tabular}

(OR: 15,3) 
Tabla 2.- Distribución de casos y controles según factor desinterés por control mensual en el programa.

\begin{tabular}{lrrr}
\hline & \multicolumn{2}{c}{ Abandono } & \\
\cline { 2 - 3 } Factor & \multicolumn{1}{c}{ Sí } & No & Total \\
\hline Positivo & 24 & 4 & 28 \\
Negativo & 102 & 248 & 350 \\
Total & 126 & 252 & 378 \\
\hline
\end{tabular}

(OR: 14,6)

El factor de inalterabilidad del estilo de vida contrario con el manejo en el Programa tuvo un OR de 4,5 (Tabla 5) y, finalmente, el factor aparición de efectos adversos de los medicamentos tuvo un OR de 1,9 (Tabla 6).

El factor maltrato del personal profesional (médico, enfermera, nutricionista, técnico de enfermería) y otros factores estudiados, como hacinamiento, incomodidad, horarios inadecuados, falta de compañía de familiar para acudir al Programa, escasos recursos económicos para traslado al Hospital, no fueron factores asociados al abandono del Programa HTA.

\section{DISCUSIÓN}

El primer factor en importancia fue el conocimiento inadecuado sobre la enfermedad. Al

Tabla 4.- Distribución de casos y controles según factor incumplimiento de tratamiento por no sentir molestias.

\begin{tabular}{|c|c|c|c|}
\hline \multirow[b]{2}{*}{ Factor } & \multicolumn{2}{|c|}{ Abandono } & \multirow[b]{2}{*}{ Total } \\
\hline & Sí & No & \\
\hline Positivo & 12 & 2 & 14 \\
\hline Negativo & 114 & 250 & 364 \\
\hline Total & 126 & 252 & 378 \\
\hline
\end{tabular}

(OR: 13,2)
Tabla 3.- Distribución de casos y controles según factor incumplimiento de tratamiento si cifras de presión arterial se normalizan.

\begin{tabular}{|c|c|c|c|}
\hline \multirow[b]{2}{*}{ Factor } & \multicolumn{2}{|c|}{ Abandono } & \multirow[b]{2}{*}{ Total } \\
\hline & Sí & No & \\
\hline Positivo & 18 & 3 & 21 \\
\hline Negativo & 108 & 249 & 357 \\
\hline Total & 126 & 252 & 358 \\
\hline
\end{tabular}

(OR: 13,8)

respecto, Paredes y colaboradores encontraron que casi uno de cada cuatro pacientes tenía conocimiento inadecuado en el Hospital Regional Honorio Delgado de Arequipa $\left({ }^{19}\right)$. Este factor tiene que ver fundamentalmente con la labor de enfermería del Programa que, durante la entrevista, deba proporcionarle toda la información necesaria para que los pacientes y familiares puedan comprender la enfermedad, conocimiento reforzado por el médico en la primera consulta.

Sin embargo, en el Programa se verifica la realización de la entrevista y también se ofrecen charlas y folletos a los pacientes y familiares. Al parecer, no se constata si éstos han logrado informarse y concientizarse debidamente o en realidad se debería cambiar de estrategias y metodologías, para llegar apropiadamente al usuario.

Tabla 5.- Distribución de casos y controles según factor inalterabilidad de estilo de vida por manejo en programa.

\begin{tabular}{|c|c|c|c|}
\hline \multirow[b]{2}{*}{ Factor } & \multicolumn{2}{|c|}{ Abandono } & \multirow[b]{2}{*}{ Total } \\
\hline & Sí & No & \\
\hline Positivo & 42 & 25 & 67 \\
\hline Negativo & 84 & 227 & 311 \\
\hline Total & 126 & 252 & 378 \\
\hline
\end{tabular}

(OR: 4,5) 
Tabla 6.- Distribución de casos y controles según factor frecuente aparición de efectos adversos.

\begin{tabular}{|c|c|c|c|}
\hline \multirow[b]{2}{*}{ Factor } & \multicolumn{2}{|c|}{ Abandono } & \multirow[b]{2}{*}{ Total } \\
\hline & Sí & No & \\
\hline Positivo & 38 & 45 & 83 \\
\hline Negativo & 88 & 207 & 295 \\
\hline Total & 126 & 252 & 378 \\
\hline
\end{tabular}

(OR: 1,9)

Otro factor que se considera de mayor riesgo para abandono de un Programa de control de Hipertensión Arterial es el de desinterés en el control mensual en el Programa, lo que sumado al incumplimiento del tratamiento antihipertensivo -sea porque las cifras de presión arterial se normalizan o porque no sienten molestias- se interpreta como parte de una conciencia inapropiada de la enfermedad, lo que coincide con Flores y Rivas $\left({ }^{20}\right)$, que hallaron que "el sentir mejoría" constituía $44,4 \%$ de las causas de abandono del tratamiento farmacológico antihipertensivo en pacientes.

Este aspecto tiene mucho que ver con la adecuada educación (concientización) del paciente, cuya responsabilidad principal está en el médico y la enfermera. Y no se trata sólo de información, pues el hipertenso debe tomar conciencia de la necesidad de modificar su estilo de vida, no interrumpir su medicación cuando la sintomatología mejore o desaparezca o las cifras de presión arterial se hallan normalizado, o no le dé la debida importancia a un control mensual en el Programa.

La inalterabilidad del cambio de estilo de vida es también un factor de riesgo que favorece el abandono del Programa, al parecer para evitar recibir reproches tanto del médico como de la enfermera o tener nuevas citas a nutricionista y consejería. Se trata, en este caso, de pacientes que tienen su punto de vista, en el sentido de que "de algo hay que morirse" y no aceptan sacrificios dietéticos o de particular manera de vivir a la que están acostumbrados.

La aparición de efectos adversos de los medicamentos tuvo un OR cercano a 2, lo que indicaba un factor de riesgo de abandono del Programa y obviamente de la medicación; es sabido que los fármacos antihipertensivos -tipo inhibidores ECA (captopril y enalapril)- pueden provocar tos; los antagonistas del calcio (nifedipino, verapamilo) producen edema de miembros inferiores y bochornos en ciertos casos.

Estos resultados difieren de los obtenidos por Flores y Rivas $\left({ }^{20}\right)$ y Paredes $\left({ }^{19}\right)$, que consideran que los efectos colaterales sólo explican entre 2 y $5 \%$ el motivo de los abandonos del tratamiento.

Por otro lado, algunos factores que fueron informados en otros estudios, como la demora en recibir la atención, el hacinamiento existente, maltrato por parte de la técnica o enfermera no han sido confirmados en la presenta investigación.

En conclusión, el conocimiento inadecuado sobre la hipertensión arterial fue el principal factor determinante del abandono del Programa de Hipertensión Arterial, en el Hospital Nacional Almanzor Aguinaga Asenjo de Essalud. Otros factores de importancia fueron los aspectos relacionados con una conciencia inadecuada de la enfermedad, que agrupa tanto al desinterés de acudir al Programa como al incumplimiento del tratamiento antihipertensivo, por normalización de las cifras de presión arterial o desaparición de síntomas. La inalterabilidad del estilo de vida y la aparición de efectos adversos de los medicamentos antihipertensivos tienen un riesgo moderado para el abandono del Programa. No se encontró asociación respecto a maltrato de personal profesional, aspectos de infraestructura (hacinamiento, incomodidad), manejo administrativo (horarios, trámites) del Programa o económicos (costos de traslado) del paciente en relación al abandono del Programa.

Como recomentaciones, se requiere que los profesionales de la salud -en especial médico y 
enfermera- brinden mayor educación al paciente y familiares, incrementando el nivel de información y concientización sobre la enfermedad y su control de por vida. Es necesario revisar e innovar estrategias y metodologías, para poder llegar con mensajes adecuados a pacientes y familiares, de manera de cambiar estilos de vida y comportamientos que favorecen el abandono de un Programa y su tratamiento, y que ponen en grave riesgo la vida y salud de los pacientes asegurados. Es importante favorecer la difusión por medios masivos de comunicación radiales, periodísticos y televisivos de información sobre la enfermedad, para que la comunidad en su conjunto le dé importancia, y así colaborar en su prevención y control.

\section{BIBLIOGRAFÍA}

1. Sosa J. Programa Nacional de Hipertensión Arterial en el IPSS. Hipertensión (Perú) 1995; 1(3): 138-40.

2. Guemez J, Alba A, Argote A, Méndez R, Moreno L. Relaciones entre peso, edad y tensión arterial en población mexicana. Arch Inst Cardiol Mex 1992; 62: 171-7.

3. Sokolow M. Cardiología Clínica. $3^{\circ}$ edición. México: Edit. El Manual Moderno, 1998.

4. Morales G. Definición y criterios de clasificación de la hipertensión arterial. Guías de tratamiento de la OMS y la Sociedad Internacional de Hipertensión. Hipertensión (Perú) 1995; 1(2): 41-50.

5. Evans R. Factores de riesgo de la cardiopatía isquémica coronaria. Rev Per Epidem 1990; 3: 25-34.

6. Oliveros J. Prevalencia de HTA en Lambayeque. Hipertensión (Perú) 1997; 3(3).

7. Soto Cáceres V, Alberca B L, Colchado J. Prevalencia de hipertensión arterial en la ciudad de Chiclayo. Libro de Resúmenes IX Congreso Nacional de Medicina Interna Lima 1996.
8. Alberca BL, Soto Cáceres V, Colchado J. Hipertensión arterial en población asegurada de la ciudad de Chiclayo. Libro de Resúmenes IX Congreso Nacional de Medicina Interna Lima 1996.

9. Samamé ZLL, Gonzáles CL. Prevalencia de hipertensión arterial en Monsefú. Tesis (Médico Cirujano) Universidad Nacional Pedro Ruiz Gallo, Lambayeque, 1996.

10. Cañizares J. Hipertensión: Un tema siempre actual. Hexágono 1992; 1(2): 5-12.

11. Sánchez Palacios P. Hipertensión arterial. VI Reporte del Comité Nacional Conjunto de los Institutos Nacionales de Salud de los Estados Unidos de América. Innovartis 1998; 1: $25-50$.

12. IPSS. Hipertensión arterial: Nuevos horizontes. Bol Terap IPSS 1993; 2(22): 1-2.

13. De los Arcos E. Hipertensión arterial. Bol Serv Cardiol Hosp Navarro España 1997; 21(1).

14. IPSS. Protocolo de Hipertensión Arterial. Lima, 1998-

15. Oficina de Epidemiología y Programas. Boletín Epidemiológico Anual 1999 Hospital Nacional “Almanzor Aguinaga Asenjo" Essalud. Mayo 2000.

16. Soto Cáceres V. Factores asociados al abandono de pacientes del Programa de Hipertensión Arterial del Hospital Nacional "Almanzor Aguinaga Asenjo" IPSS Chiclayo. Libro de Resúmenes IX Congreso Nacional de Medicina Interna, Lima 1998.

17. Joint National Committee on Detection, Evaluation, and Treatment of High Blood Pressure. The sixth report (JNC VI). Arch Intern Med 1997; 157: 2413-46.

18. WHO.1999 World Health Organization International Society of Hypertension guidelines for the management of hypertension. J Hypert 1999; 17:151-83.

19. Paredes JA, Sanz MG, Bottazi AR, Murillo VR, Anci TM. Estudio clínico epidemiológico de la hipertensión arterial en el Hospital Regional "Honorio Delgado", Arequipa-Perú. Hipertensión (Perú) 1997; 3(3).

20. Flores TR, Rivas VJ. Prevalencia de diabetes mellitus, obesidad, hipertensión arterial, hipercolesterolemia, como factores de riesgo de enfermedad cardiovascular y cerebrovascular en la población adulta del distrito de Chiclayo. Febrero-Abril 1995. Tesis (Medico Cirujano) Universidad Nacional Pedro Ruiz Gallo Lambayeque, 1995. 